\title{
Homotopy Perturbation Transform Method for Solving Third Order Korteweg-DeVries (KDV) Equation
}

\author{
Abdelilah Kamal Hassan Sedeeg ${ }^{1,2}$ \\ ${ }^{1}$ Mathematics Department Faculty of Sciences and Arts, Almikwah-Albaha University, Albaha, Saudi Arabia \\ ${ }^{2}$ Mathematics Department Faculty of Education, Holy Quran and Islamic Sciences University, Khartoum, Sudan
}

Email address:

aelilah63@hotmail.com

To cite this article:

Abdelilah Kamal Hassan Sedeeg. Homotopy Perturbation Transform Method for Solving Third Order Korteweg-DeVries (KDV) Equation. American Journal of Applied Mathematics. Vol. 4, No. 5, 2016, pp. 247-251. doi: 10.11648/j.ajam.20160405.16

Received: September 4, 2016; Accepted: September 26, 2016; Published: October 18, 2016

\begin{abstract}
In this paper, we develop a method to calculate approximate solution of some Third-order Korteweg-de Vries equations with initial condition with the help of a new method called Aboodh transform homotopy perturbation method (ETHPM). This method is a combination of the new integral transform "Aboodh transform" and the homotopy perturbation method. The nonlinear term can be easily handled by homotopy perturbation method. The results reveal that the combination of Aboodh transform and homotopy perturbation method is quite capable, practically well appropriate for use in such problems and can be applied to other nonlinear problems. This method is seen as a better alternative method to some existing techniques for such realistic problems.
\end{abstract}

Keywords: Aboodh Transform, Homotopy Perturbation Method, Korteweg-DeVries (KDV) Equation

\section{Introduction}

The exact solutions of nonlinear partial differential equations are difficult, therefore many various approximate methods have recently been developed such as homotopy perturbation method [1-6], Adomian's decomposition method, differential transform method and projected differential transform method to solve linear and nonlinear differential equations. The homotopy perturbation method has the merits of simplicity and easy execution. Homotopy theory becomes a powerful mathematical tool also ; homotopy theory can overcome the difficulties arising in calculation of Adomian's polynomials in Adomian's decomposition method. The KDV equation plays an important role in diverse areas of engineering and scientific applications, and therefore, the enormous amount of research work has been invested in the study of KDV equations [7-12]. The Aboodh transform [13$16]$ is totally incapable of handling nonlinear equations because of the difficulties that are caused by the nonlinear terms. Furthermore, the homotopy perturbation method is also combined with the well-known Aboodh transform method and the variational iteration method to produce a highly effective technique for handling many nonlinear problems.

In this paper, we shall deal with the KDV equation in the following form,

$$
u_{t}+a u u_{x}+b u_{x x x}=0
$$

Where $u(x, t)$ is the displacement.KDV equation Was first derived by Korteweg and Vries (1895) to the water waves in shallow canal, when the study of water waves was of vital interest for applications in naval architecture and for the knowledge of tides and floods. The purpose of this paper is to extend the (HPTM) for the solution of Korteweg-DeVries (KDV) Equation. The method has been successfully applied for obtaining exact solutions for nonlinear equations.

\section{Aboodh Transform}

\section{Definition:}

A new transform called the Aboodh transform defined for function of exponential order we consider functions in the set a, defined by:

$$
A=\left\{f(t): \exists M, k_{1}, k_{2}>0,|f(t)|<M e^{-v t}\right.
$$

For a given function in the set $\mathrm{M}$ must be finite 
number, $k_{1}, k_{2}$ may be finite or infinite.Aboodh transform which is defined by the integral equation

$$
A[f(t)]=K(v)=\frac{1}{v} \int_{0}^{\infty} f(t) e^{-v t} d t, t \geq 0, \mathrm{k}_{1} \leq v \leq \mathrm{k}_{2}(2)
$$

Aboodh transform of partial derivative:

To obtain Aboodh transform of partial derivative we use integration by parts, and then we have:

$$
\begin{gathered}
A\left[\frac{\partial u(x, t)}{\partial t}\right]=v K(x, v)-\frac{u(x, 0)}{v}, \\
A\left[\frac{\partial^{2} u(x, t)}{\partial t^{2}}\right]=v^{2} K(x, v)-\frac{1}{v} \frac{\partial u(x, 0)}{\partial t}-u(x, 0)
\end{gathered}
$$

Proof: To obtain transforms of partial derivatives we use integration by parts as follows:

$$
\begin{gathered}
A\left[\frac{\partial u(x, t)}{\partial t}\right]=\frac{1}{v} \int_{0}^{\infty} \frac{\partial u(x, t)}{\partial t} e^{-v t} d t= \\
\lim _{p \rightarrow \infty} \frac{1}{v} \int_{0}^{p} \frac{\partial u(x, t)}{\partial t} e^{-v t} d t=\lim _{p \rightarrow \infty}\left\{\left[\frac{1}{v} u(x, t) e^{-v t}\right]_{0}^{p}+\right. \\
\left.\frac{1}{v} \int_{0}^{p} u(x, t) e^{-v t} d t\right\}=v K(x, v)-\frac{u(x, 0)}{v}(3)
\end{gathered}
$$

We assume that $f$ is piecewise continuous and it is of exponential order.

Let $\frac{\partial u(x, t)}{\partial t}=g$ then, by using Eq. (2) we have

$$
\begin{array}{r}
A\left[\frac{\partial^{2} u(x, t)}{\partial t^{2}}\right]=A\left[\frac{\partial g(x, t)}{\partial t}\right]=v A(g(x, t))-\frac{g(x, 0)}{v}= \\
v^{2} K(x, v)-\frac{1}{v} \frac{\partial u(x, 0)}{\partial t}-u(x, 0)(4)
\end{array}
$$

We can easily extend this result to the $n t h$ partial derivative by using mathematical induction.

\section{Homotopy Perturbation Method}

Let $X$ and $Y$ be the topological spaces. If $f$ and $g$ are continuous maps of the space $X$ into $Y$, it is said that $f$ is homotopic to $g$, if thereis continuous $\operatorname{map} F: X \times[0,1] \rightarrow$ $Y$ such that $F(x, 0)=f(x)$ and $F(x, 1)=g(x)$, foreach $x \in$ $X$,then the map is called homotopy between $f$ and $g$.

To explain the homotopy perturbation method, we consider a general equation of the type,

$$
L(U)=0
$$

Where $L$ is any differential operator, we define a convex homotopy $H(U, p)$ by

$$
H(U, p)=(1-p) F(U)+p L(U)
$$

Where $F(U)$ is a functional operator with known solution $V_{0}$ which can be obtained easily. It is clear that, for

$$
H(U, p)=0
$$

We have: $H(U, 0)=F(U), H(U, 1)=L(U)$.

In topology this show that $H(U, P)$ continuously traces an implicitly defined carves from a starting point $H\left(V_{0}, 0\right)$ to a solution function $H(f, 1)$. The HPM uses the embed ling parameter $p$ as a small parameter and write the solution as a power series

$$
U=U_{0}+p U_{1}+p^{2} U_{2}+p^{3} U_{3}+\ldots
$$

If $p \rightarrow 1$, then Eq. (8) corresponds to Eq. (6) and becomes the approximate solution of the form,

$$
f=\lim _{p \rightarrow 1} U=\sum_{i=0}^{\infty} U_{i}
$$

We assume that Eq. (8) has a unique solution. The comparisons of like powers of $p$ give solutions of various orders, for more details see [17-20].

\subsection{Basic Idea}

To illustrate the basic idea of this method, we consider a general form of nonlinear non homogeneous partial differential equation as the follow:

$$
D u(x, t)+R u(x, t)+N u(x, t)=g(x, t)
$$

with the following initial conditions

$$
u(x, 0)=h(x), u_{t}(x, 0)=f(x)
$$

Where $\mathrm{D}$ is the second order linear differential operator $D=\frac{\partial^{2}}{\partial t^{2}}$, is the linear differential operator ofless order than $\mathrm{D}$, $\mathrm{N}$ represents the general non-linear differential operator and $g(x, t)$ is the source term.

Taking Aboodh transform (denoted throughout this paper $\operatorname{by} A()$.$) on both sides of Eq. (10), to get:$

$$
A[D u(x, t)]+A[R u(x, t)]+A[N u(x, t)]=A[g(x, t)](1
$$

Using the differentiation property of Aboodh transform and above initial conditions, we have:

$$
\begin{array}{r}
A[u(x, t)]=\frac{1}{v^{2}} A[g(x, t)]+\frac{1}{v^{2}} h(x)+\frac{1}{v^{3}} f(x)- \\
\frac{1}{v^{2}} A[R u(x, t)+N u(x, t)](13)
\end{array}
$$

Operating with the Aboodh inverse on both sides of Eq. (12) gives:

$$
u(x, t)=G(x, t)-A^{-1}\left[\frac{1}{v^{2}} A[R u(x, t)+N u(x, t)]\right](14
$$

Where $G(x, t)$ represents the term arising from the source term and the prescribed initial condition. Now, we apply the homotopy perturbation method

$$
u(x, t)=\sum_{n=0}^{\infty} p^{n} u_{n}(x, t)
$$

And the nonlinear term can be decomposed as:

$$
N u(x, t)=\sum_{n=0}^{\infty} p^{n} H_{n}(u)
$$

Where $H_{n}(u)$ are He's polynomial and given by:

$H_{n}\left(u_{0}, u_{1}, u_{2} \ldots u_{n}\right)=\frac{1}{n !} \frac{\partial^{n}}{\partial p^{n}}\left[N\left(\sum_{n=0}^{\infty} p^{i} u_{i}\right)\right]_{p=0}, n=0,1,2, \ldots$

Substituting Eqs. (15) and (16) in Eq. (14) we get: 


$$
\begin{array}{r}
\sum_{n=0}^{\infty} p^{n} u_{n}(x, t)=G(x, t)-p A^{-1}\left[\frac { 1 } { v ^ { 2 } } A \left[R \sum_{n=0}^{\infty} p^{n} u_{n}(x, t)+\right.\right. \\
\left.\left.\sum_{n=0}^{\infty} p^{n} H_{n}(u)\right]\right](18)
\end{array}
$$

Which is the coupling of the Aboodh transform and the homotopy perturbation method using He's polynomials. Comparing the coefficient of same powers of $\mathrm{p}$, thefollowingapproximations are obtained:

$$
\begin{gathered}
p^{0}: u_{0}(x, t)=G(x, t), \\
p^{1}: u_{1}(x, t)=-A^{-1}\left[\frac{1}{v^{2}} A\left[R u_{0}(x, t)+H_{0}(u)\right],\right. \\
p^{2}: u_{2}(x, t)=-A^{-1}\left[\frac{1}{v^{2}} A\left[R u_{1}(x, t)+H_{1}(u)\right]\right], \\
p^{3}: u_{3}(x, t)=-A^{-1}\left[\frac{1}{v^{2}} A\left[R u_{2}(x, t)+H_{2}(u)\right]\right],
\end{gathered}
$$

Then the solution is;

$$
u(x, t)=\lim _{p \rightarrow 1} u_{n}(x, t)=u_{0}+u_{1}+u_{2}+\cdots
$$

\subsection{Applications}

In this section, the effectiveness and the usefulness of homotopy perturbation transform method (HPTM) are demonstrated by finding exact solutions of KortewegDeVries (KDV) Equation.

Example 3.1.

Consider the following linear homogeneous KDV Equation;

$$
u_{t}-6 u u_{x}+u_{x x x}=0
$$

With the initial condition;

$$
u(x, 0)=6 x
$$

Applying the Aboodh transform of both sides of Eq. (20),

$$
A\left[u_{t}\right]=-A\left[u_{x x x}-6 u u_{x}\right]
$$

Using the differential property of Aboodh transform Eq. (22) can be written as:

$$
v A[u(x, t)]-\frac{1}{v} u(x, 0)=-A\left[u_{x x x}-6 u u_{x}\right]
$$

Using initial condition Eq. (21), Eq. (23) can be written as:

$$
A[u(x, t)]=\frac{6 x}{v^{2}}-\left[\frac{1}{v} A\left[u_{x x x}-6 u u_{x}\right]\right]
$$

The inverse Aboodh transform implies that:

$$
u(x, t)=6 x-A^{-1}\left[\frac{1}{v} A\left[u_{x x x}-6 u u_{x}\right]\right]
$$

Now, we apply the homotopy perturbation method, we get:

$$
\begin{array}{r}
\sum_{n=0}^{\infty} p^{n} u_{n}(x, t)=6 x-p A^{-1}\left[\frac{1}{v} A\left(\sum_{n=0}^{\infty} p^{n} u_{n}(x, t)\right)_{x x x}-\right. \\
\left.\sum_{n=0}^{\infty} p^{n} H_{n}(u)\right](26)
\end{array}
$$

Where $H_{n}(u)$ are He's polynomials that represent the nonlinear terms.

The first few components of He's polynomials are given by

$$
\begin{gathered}
H_{0}(u)=u_{0} u_{0 x} \\
H_{1}(u)=u_{0} u_{1 x}+u_{1} u_{0 x} \\
H_{2}(u)=u_{0} u_{2 x}+u_{1} u_{1 x}+u_{2} u_{0 x}
\end{gathered}
$$

Comparing the coefficient of same powers of $p$, the following approximations are obtained ;

$$
\begin{aligned}
& p^{0}: u_{0}(x, t)=6 x \\
p^{1}: u_{1}(x, t)= & -A^{-1}\left[\frac{1}{v} A\left[u_{0}(x, t)_{x x x}-6 H_{0}(u)\right]\right] \\
= & -A^{-1}\left[\frac{1}{v} A\left[-6 u_{0} u_{0 x}\right]\right] \\
= & -A^{-1}\left[\frac{1}{v} A[-6(6 x)(6)]\right] \\
= & -A^{-1}\left[-\frac{6^{3} x}{v^{3}}\right]=6^{3} x t \\
p^{2}: u_{2}(x, t)= & -A^{-1}\left[\frac{1}{v} A\left[u_{1}(x, t)_{x x x}-6 H_{1}(u)\right]\right] \\
= & -A^{-1}\left[\frac{1}{v} A\left[-6\left(u_{0} u_{1 x}+u_{1} u_{0 x}\right)\right]\right] \\
= & -A^{-1}\left[\frac{1}{v} A\left[-6\left(6^{4} x t+6^{4} x t\right)\right]\right] \\
= & -A^{-1}\left[-2 \frac{6^{5} x}{v^{4}}\right]=6^{5} x t^{2}
\end{aligned}
$$

$p^{3}: u_{3}(x, t)=-A^{-1}\left[\frac{1}{v} A\left[-6\left(u_{0} u_{2 x}+u_{1} u_{1 x}+u_{2} u_{0 x}\right)\right]\right]$ $=6^{7} x t^{3}$

Therefore the solution $u(x, t)$ is given by:

$$
u(x, t)=6 x\left(1+36 t+(36 t)^{2}+(36 t)^{3}+\cdots\right)
$$

In series form,

$$
u(x, t)=\frac{6 x}{1-36 t},|36 t|<1
$$

Example 3.2.

Consider the following linear homogeneous KDV Equation;

$$
u_{t}+u u_{x}+u_{x x x}=0
$$

With the initial condition;

$$
u(x, 0)=1-x
$$

Applying the Aboodh transform of both sides of Eq. (29),

$$
A\left[u_{t}\right]=-A\left[u_{x x x}+u u_{x}\right]
$$

Using the differential property of Aboodh transform Eq.(31) can be written as: 


$$
v A[u(x, t)]-\frac{1}{v} u(x, 0)=-A\left[u_{x x x}+u u_{x}\right]
$$

Using initial condition Eq. (30), Eq. (32) can be written as:

$$
A[u(x, t)]=\frac{1-x}{v^{2}}-\left[\frac{1}{v} A\left[u_{x x x}+u u_{x}\right]\right]
$$

The inverse Aboodh transform implies that:

$$
u(x, t)=1-x-A^{-1}\left[\frac{1}{v} A\left[u_{x x x}+u u_{x}\right]\right]
$$

Now, we apply the homotopy perturbation method, we get:

$$
\begin{array}{r}
\sum_{n=0}^{\infty} p^{n} u_{n}(x, t)=1-x-p A^{-1}\left[\frac{1}{v} A\left(\sum_{n=0}^{\infty} p^{n} u_{n}(x, t)\right)_{x x x}+\right. \\
\left.\sum_{n=0}^{\infty} p^{n} H_{n}(u)\right](35)
\end{array}
$$

Comparing the coefficient of same powers of $p$, the following approximations are obtained:

$$
\begin{aligned}
& p^{0}: u_{0}(x, t)=1-x \\
p^{1}: u_{1}(x, t) & =-A^{-1}\left[\frac{1}{v} A\left[u_{0}(x, t)_{x x x}+H_{0}(u)\right]\right] \\
& =-A^{-1}\left[\frac{1}{v} A\left[u_{0} u_{0 x}\right]\right] \\
& =-A^{-1}\left[\frac{1}{v} A[x-1]\right]=-A^{-1}\left[\frac{(x-1)}{v^{3}}\right] \\
& =(1-x) t \\
p^{2}: u_{2}(x, t) & =-A^{-1}\left[\frac{1}{v} A\left[u_{1}(x, t)_{x x x}+H_{1}(u)\right]\right] \\
& =-A^{-1}\left[\frac{1}{v} A\left[\left(u_{0} u_{1 x}+u_{1} u_{0 x}\right)\right]\right] \\
& \left.=-A^{-1}\left[\frac{1}{v} A[(x-1) t+(x-1) t)\right]\right] \\
& =-A^{-1}\left[2 \frac{(x-1)}{v^{4}}\right]=(1-x) t^{2} \\
p^{3}: u_{3}(x, t)= & -A^{-1}\left[\frac{1}{v} A\left[\left(u_{0} u_{2 x}+u_{1} u_{1 x}+u_{2} u_{0 x}\right)\right]\right] \\
= & -A^{-1}\left[\frac{1}{v}\left[6 \frac{(x-1)}{v^{5}}\right]\right]=(1-x) t^{3}
\end{aligned}
$$

Therefore the solution $u(x, t)$ is given by:

$$
u(x, t)=(1-x)\left(1+t+t^{2}+t^{3}+\cdots\right)
$$

In series form,

$$
u(x, t)=\frac{1-x}{1-t}
$$

Example 3.3.

Consider the following linear homogeneous KDV Equation;

$$
u_{t}-6 u u_{x}+u_{x x x}=0
$$

With the initial condition,

$$
u(x, 0)=-2 \frac{k^{2} e^{k x}}{\left(1+e^{k x}\right)^{2}}
$$

Applying the Aboodh transform of both sides of Eq. (38),

$$
A\left[u_{t}\right]=-A\left[u_{x x x}-6 u u_{x}\right]
$$

Using the differential property of Aboodh transform Eq. (40) can be written as:

$$
v A[u(x, t)]-\frac{1}{v} u(x, 0)=-A\left[u_{x x x}-6 u u_{x}\right]
$$

Using initial condition Eq. (39), Eq. (41) can be written as:

$$
A[u(x, t)]=\frac{-2 \frac{k^{2} e^{k x}}{\left(1+e^{k x}\right)^{2}}}{v^{2}}-\left[\frac{1}{v} A\left[u_{x x x}-6 u u_{x}\right]\right]
$$

The inverse Aboodh transform implies that:

$$
u(x, t)=-2 \frac{k^{2} e^{k x}}{\left(1+e^{k x}\right)^{2}}-A^{-1}\left[\frac{1}{v} A\left[u_{x x x}-6 u u_{x}\right]\right]
$$

Now, we apply the homotopy perturbation method, we get:

$$
\begin{aligned}
& \sum_{n=0}^{\infty} p^{n} u_{n}(x, t)=-2 \frac{k^{2} e^{k x}}{\left(1+e^{k x}\right)^{2}}- \\
& \quad p A^{-1}\left[\frac{1}{v} A\left(\sum_{n=0}^{\infty} p^{n} u_{n}(x, t)\right)_{x x x}-\sum_{n=0}^{\infty} p^{n} H_{n}(u)\right]
\end{aligned}
$$

Comparing the coefficient of same powers of $p$, the following approximations are obtained:

$$
\begin{gathered}
p^{0}: u_{0}(x, t)=-2 \frac{k^{2} e^{k x}}{\left(1+e^{k x}\right)^{2}} \\
p^{1}: u_{1}(x, t)=-2 \frac{k^{5} e^{k x}\left(e^{k x}-1\right)}{\left(1+e^{k x}\right)^{3}} t \\
p^{2}: u_{2}(x, t)=-2 \frac{k^{8} e^{k x}\left(e^{2 k x}-4 e^{k x}+1\right)}{\left(1+e^{k x}\right)^{4}} t^{2}
\end{gathered}
$$

Therefore the solution $u(x, t)$ is given by:

$$
u(x, t)=-2 \frac{k^{2} e^{k x}}{\left(1+e^{k x}\right)^{2}}-2 \frac{k^{5} e^{k x}\left(e^{k x}-1\right)}{\left(1+e^{k x}\right)^{3}} t-
$$

$$
2 \frac{k^{8} e^{k x}\left(e^{2 k x}-4 e^{k x}+1\right)}{\left(1+e^{k x}\right)^{4}} t^{2}-
$$

In series form,

$$
u(x, t)=-2 \frac{k^{2} e^{k\left(x-k^{2} t\right)}}{\left(1+e^{k\left(x-k^{2} t\right)}\right)^{2}}
$$

\section{Conclusions}

In this paper, we have applied the homotopy perturbation transform method to Korteweg-DeVries (KDV) Equation. It can be concluded that the HPTM is a very powerful and efficient technique in finding exact and approximates solutions for nonlinear problems. By using this method we 
obtain a new efficient recurrent relation to solve (KDV) Equation.

\section{References}

[1] J. H. He, Homotopy perturbation technique, Computer Methods in Applied Mechanics and Engineering, 178 (1999):257-262.

[2] N. H. Sweilam and M.M. Khader, Exact solutions of some coupled nonlinear partial differential equations using the homotopy perturbation method, Computers \& Mathematics with Applications, 58 (2009):2134 2141.

[3] A.M. Wazwas, A study on linear and non-linear Schrodinger equations by the variational iteration method, Chaos, Solitions and Fractals, 37 (4) (2008):1136 1142.

[4] B. Jazbi and M. Moini, Application of He's homotopy perturbation method for Schrodinger equation, Iranian Journalof Mathematical Sciences and Informatics, 3 (2) (2008):13-19.

[5] J. H. He, Homotopy perturbation method: a new nonlinear analytical technique, Applied Mathematics and Computa- tion, 135 (2003):73-79.

[6] J. H. He, Comparison of homotopy perturbation method and homotopy analysis method, Applied Mathematics and Computation, 156 (2004):527539.

[7] Dogan Kaya, Mohammed Aassila, Application for a generalized $\mathrm{KdV}$ equation by the decomposition method, Physics Letters A 299 (2002) 201-206.

[8] P. G. Drazin, R. S. Johnson, Solutions: An Introduction, Cambridge University Press, Cambridge, 1989.

[9] P. Saucez, A. V. Wouwer, W. E. Schiesser, An adaptive method of lines solution of the Korteweg-de Vries equation, Computers \& Mathematics with Applications 35 (12) (1998) 13-25.
[10] T. A. Abassy, Magdy A. El-Tawil, H. El-Zoheiry, Exact solutions of some nonlinear partial differential equations using the variational iteration method linked with Laplace transforms and the Pad'e technique, Computers and Mathematics with Applications, doi:10.1016/j.camwa.2006.12.067.

[11] F. Kangalgil, F. Ayaz, Solitary wave solutions for the KdV and $\mathrm{KdV}$ equations by differential transform method, Chaos, Solitons and Fractals, doi:10.1016/j.chaos.2008.02.009.

[12] Mohannad H. Eljaily1, Tarig M. Elzaki, Homotopy Perturbation Transform Method for Solving KortewegDeVries (KDV) Equation, Pure and Applied Mathematics Journal 2015; 4 (6): 264-268.

[13] K. S. Aboodh, The New Integral Transform "Aboodh Transform" Global Journal of pure and Applied Mathematics, 9 (1), 35-43 (2013).

[14] K. S. Aboodh, Application of New Transform "Aboodh transform" to Partial Differential Equations, Global Journal of pure and Applied Math, 10 (2),249-254 (2014).

[15] Khalid Suliman Aboodh, Homotopy Perturbation Method and Aboodh Transform for Solving Nonlinear Partial Differential Equations, Pure and Applied Mathematics Journal Volume 4, Issue 5, October 2015, Pages: 219-224.

[16] Khalid Suliman Aboodh, Solving Fourth Order Parabolic PDE with Variable Coefficients Using Aboodh Transform Homotopy Perturbation Method, Pure and Applied Mathematics Journal 2015; 4 (5): 219-224.

[17] L. Debnath, Nonlinear Partial Differential Equations for Scientists and Engineers, Birkhauser, Bostn, 1997.

[18] J. D. Logan, An Introduction to Nonlinear Partial Differential Equations, Wiley, New York, 1994.

[19] G. B. Whitham, Linear and Nonlinear Waves, Wiley, New York, 1974.

[20] Sweilam, N. H. and M.M. Khader, 2009. Exact Solutions of some Capled nonlinear 